\title{
CRITERIA FOR EXTREME FORMS
}

\author{
E. S. BARNES
}

(rec. 8 Aug. 1958)

1. A positive quadratic form $f(x)=\sum_{i, j=1}^{n} a_{i j} x_{i} x_{j}\left(a_{i j}=a_{j i}\right)$, of determinant $\left\|a_{i j}\right\|=D$ and minimum $M$ for integral $x \neq 0$, is said to be extreme if the ratio $M / D^{1 / n}$ is a (local) maximum for small variations in the coefficients $a_{i j}$.

Minkowski [3] has given a criterion for extreme forms in terms of a fundamental region (polyhedral cone) in the coefficient space. This criterion, however, involves a complete knowledge of the edges of the region and is therefore of only theoretical value.

Voronoï [4] has given the only practical criterion in:

THEOREM 1. A positive quadratic form is extreme if and only if it is perfect and eutactic.

I have recently established, in [1], a criterion in terms of linear inequalities and shown how Theorem 1 may be simply deduced from it:

THEOREM 2. If $f$ has minimal vectors $\pm m_{1}, \cdots, \pm m_{s}$, then it is extreme if and only if there exists no non-trivial quadratic form $g(x)=\sum_{i, j=1}^{n} b_{i j} x_{i} x_{j}$ satisfying

$$
g\left(m_{k}\right) \geqq 0 \quad(k=1, \cdots, s), \sum_{i, j=1}^{n} A_{i j} b_{i j} \leqq 0,
$$

where $F(x)=\Sigma A_{i j} x_{i} x_{j}$ is the adjoint of $f(x)$.

I give here two further criteria, in Theorems 3 and 4 . Theorem 3 amounts to a refinement of Theorem 1 in terms of a subset of the minimal vectors. It has the important practical consequences that, in general, (i) only a suitable subset of the minimal vectors need be specified or even known; and (ii) the calculations required to check that a form is eutactic are considerably simplified.

Theorem 4 shows further that the eutactic condition may sometimes be replaced by a simple condition on the group of automorphs of the form.

2. The minimal vectors of $f$ are defined to be the integral solutions $x= \pm m_{1}, \cdots, \pm m_{s}$ of $f(x)=M$. Let $H$ be any subset of the minimal vectors, say $\pm m_{1}, \cdots, \pm m_{t}(t \leqq s)$. We shall say that $f$ is $H$-perfect if 
$f$ is uniquely determined by $H$ and its minimum $M$; i.e. if there exists no non-trivial quadratic form $g(x)$ satisfying

$$
g\left(m_{k}\right)=0 \quad(k=1, \cdots, t) .
$$

If $F(x)=\Sigma A_{i j} x_{i} x_{j}$ is the adjoint of $f(x)$, we shall say that $f$ is $H$-eutactic if $F(x)$ is expressible as

$$
F(x) \equiv \sum_{k=1}^{t} \rho_{k}\left(m_{k}^{\prime} x\right)^{2} \text { with } \rho_{k}>0 \quad(k=1, \cdots, t) .
$$

These definitions reduce to the accepted definitions of the terms perfect and eutactic if $H$ is the set of all minimal vectors.

THEOREM 3. $f$ is extreme if and only if there exists a subset $H$ of its minimal vectors such that $f$ is $H$-perfect and $H$-eutactic.

Proof. (i) The necessity of the condition is contained in Voronoïs Theorem 1, with $H$ the set of all minimal vectors.

(ii) Suppose that $f$ is $H$-perfect and $H$-eutactic, where $H=\left\{m_{1}, \cdots, m_{t}\right\}$. It then follows that a quadratic form $g(x)=\Sigma b_{i j} x_{i} x_{j}$ satisfying

$$
g\left(m_{k}\right) \geqq 0 \quad(k=1, \cdots, t), \quad \Sigma A_{i j} b_{i j} \leqq 0
$$

is necessarily trivial. For, choosing $\rho_{k}>0$ to satisfy (3), we have

$$
\begin{gathered}
A_{i j}=\sum_{k=1}^{t} \rho_{k} m_{k i} m_{k j} \quad(i, j=1, \cdots, n), \\
\Sigma A_{i j} b_{i j}=\sum_{k=1}^{t} \rho_{k} g\left(m_{k}\right) ;
\end{gathered}
$$

since $\rho_{k}>0$, the relations (4) show at once that

$$
g\left(m_{k}\right)=0 \quad(k=1, \cdots, t),
$$

whence $g(x) \equiv 0$, since $f$ is $H$-perfect.

It follows that, a fortiori, the inequalities (1) have no non-trivial solution. Hence, by Theorem $2, f$ is extreme.

3. Let $\boldsymbol{G}$ be the group of automorphs of $f$, i.e. the set of integral unimodular transformations $T$ satisfying $f(T x)=f(x)$. If $m$ is a minimal vector of $f$, then so also is $T m$; thus $\boldsymbol{G}$ may be regarded as a permutation group on the minimal vectors.

THEOREM 4. Suppose that there exists a subset $H$ of the minimal vectors of $f$ such that $f$ is $H$-perfect and $\boldsymbol{G}$ is transitive on $H$. Then $f$ is extreme.

Proof. Since $\boldsymbol{G}$ is transitive on $H, H$ is contained in a unique system of transitivity of $\boldsymbol{G}$, say $K=\left\{m_{1}, \cdots, m_{t}\right\}$. Since $f$ is $H$-perfect, it is $K$ perfect, and so the equations

$$
\sum_{i, j=1}^{n} b_{i j} m_{k i} m_{k j}=0 \quad(k=1, \cdots, t), \quad\left(b_{i j}=b_{j i}\right)
$$


have the unique solution $b_{i j}=0$. The $t \times \frac{1}{2} n(n+1)$ matrix $\left(m_{k i} m_{k j}\right)$ therefore has rank $\frac{1}{2} n(n+1)$, so that the equations

$$
\sum_{k=1}^{t} \sigma_{k} m_{k i} m_{k j}=A_{i j} \quad(i, j=1, \cdots, n)
$$

certainly possess a solution $\sigma_{1}, \cdots, \sigma_{t}$. For any such solution, we have

$$
F(x)=\Sigma A_{i j} x_{i} x_{j}=\sum_{k=1}^{t} \sigma_{k}\left(m_{k}^{\prime} x\right)^{2} .
$$

Let now $\boldsymbol{G}^{\prime}$ be the group of automorphs of $F(x)$, so that $T \in \boldsymbol{G}^{\prime}$ if and only if $T^{\prime-1} \in \boldsymbol{G}$. $\boldsymbol{G}^{\prime}$ may be interpreted as a permutation group on the linear forms $m_{k}^{\prime} x$, wherein the set $\left\{m_{1}^{\prime} x, \cdots, m_{t}^{\prime} x\right\}$ now forms a system of transitivity. Hence, if $\boldsymbol{G}^{\prime}$ has order $g$, there are precisely $g / t$ elements of $\boldsymbol{G}^{\prime}$ transforming any one form of this set into any other. Applying all the transformations of $\boldsymbol{G}^{\prime}$ to (5), and adding, we therefore obtain

Thus

$$
g F(x)=\frac{g}{t} \sum_{k=1}^{t}\left(\sigma_{1}+\sigma_{2}+\cdots+\sigma_{t}\right)\left(m_{k}^{\prime} x\right)^{2}
$$

$$
F(x)=\rho \sum_{k=1}^{t}\left(m_{k}^{\prime} x\right)^{2}, \quad \rho=\frac{1}{t}\left(\sigma_{1}+\cdots+\sigma_{t}\right)
$$

where clearly $\rho>0$ since $F$ is positive definite.

$f$ is therefore $K$-eutactic, and Theorem 3 shows now that $f$ is extreme.

4. It is perhaps worth noting that Theorem 3 would become false if stated in the stronger form: 'If $H$ is a subset of the minimal vectors of $f$ such that $f$ is $H$-perfect, then $f$ is extreme if and only if it is $H$-eutactic.' A simple counter-example is the extreme form $B_{n}$ (in the notation of [2]) defined by

$$
f(x)=\sum_{1}^{n} x_{i}^{2}
$$

with the lattice of integral $x$ satisfying

$$
\sum_{1}^{n} x_{i} \equiv 0 \quad(\bmod 2) \text {. }
$$

Here $D=4, M=2$, and the $n(n-1)$ pairs of minimal vectors are given by $m=e_{i} \pm e_{j}(i<j)$ (where $e_{i}$ is the $i$-th unit vector).

There are clearly proper subsets $H$ for which $f$ is $H$-perfect (and also proper subsets $H$ for which $f$ is $H$-eutactic). However, suppose that $f$ is both $H$-perfect and $H$-eutactic, and consider any fixed pair of suffixes $i, j(i<j)$. $H$ must contain at least one of $e_{i} \pm e_{j}$, else (2) could be satisfied by an arbitrary choice of $b_{i j}$. Also, in any relation of the type 


$$
F(x)=\sum_{1}^{n} x_{i}^{2}=\Sigma \rho_{i j}\left(x_{i}+x_{j}\right)^{2}+\Sigma \sigma_{i j}\left(x_{i}-x_{j}\right)^{2}
$$

we have $\rho_{i j}-\sigma_{i j}=0$; hence, since $f$ is $H$-eutactic, $H$ must contain neither or both of the vectors $e_{i} \pm e_{j}$. It follows that $H$ contains both $e_{i} \pm e_{j}$, for all $i<j$, so that $H$ is the complete set of minimal vectors.

It is not difficult to show also that the converse of Theorem 4 is false. The form defined by

$$
f(x)=\sum^{9} x_{i}^{2}
$$

with the lattice of integral $x$ satisfying

$$
x_{1} \equiv x_{2} \equiv \cdots \equiv x_{8} \quad(\bmod 2), \sum_{1}^{9} x_{i} \equiv 0 \quad(\bmod 4),
$$

has in fact no set $H$ of minimal vectors satisfying the conditions of Theorem 4. However, it is easily seen to be extreme (with $M=8$ ) by applying Theorem 3 to the subset $H$ of minimal vectors $2 e_{i} \pm 2 e_{j}(1 \leqq i<j \leqq 9)$.

5. I should like to take this opportunity of correcting an error of detail in [1] which was pointed out to me by Mr. A. L. Duquette of Illinois. The equation (7) of [1] implies that $A^{-1} B$ is symmetric, and this is not necessarily true. The proof as given becomes correct if we define $C=T^{\prime} B T$, where $T$ is chosen so that $T^{\prime} A T=I$.

\section{References}

[1] Barnes, E. S., "On a theorem of Voronoi", Proc. Camb. Phil. Soc. 53 (1957), 537-539.

[2] Coxeter, H. S. M., "Extreme forms", Canad. J. Math. 3 (1951), 391-441.

[3] Minkowski, H., "Diskontinuitätsbereich für arithmetische Äquivalenz", J. reine angew. Math. 129 (1905), 220-274.

[4] Voronoï, G., "Sur quelques propriétés des formes quadratiques positive parfaites", $J$. reine angew. Math. 133 (1907), 97-178.

University of Adelaide 